\title{
On $C^{1+\alpha}$ regularity of solutions of Isaacs parabolic equations with VMO coefficients
}

\author{
N. V. Krylov
}

\begin{abstract}
We prove that boundary value problems for fully nonlinear second-order parabolic equations admit $L_{p}$-viscosity solutions, which are in $C^{1+\alpha}$ for an $\alpha \in(0,1)$. The equations have a special structure that the "main" part containing only second-order derivatives is given by a positive homogeneous function of second-order derivatives and as a function of independent variables it is measurable in the time variable and, so to speak, VMO in spatial variables.
\end{abstract}

Mathematics Subject Classification (1991). 35B65, 35D40.

Keywords. Fully nonlinear equations, Hölder regularity of derivatives, Viscosity solutions.

\section{Introduction}

In this article we take a function $H(u, t, x)$,

$$
u=\left(u^{\prime}, u^{\prime \prime}\right), \quad u^{\prime}=\left(u_{0}^{\prime}, u_{1}^{\prime}, \ldots, u_{d}^{\prime}\right) \in \mathbb{R}^{d+1}, \quad u^{\prime \prime} \in \mathbb{S}, \quad(t, x) \in \mathbb{R}^{d+1},
$$

where $\mathbb{S}$ is the set of symmetric $d \times d$ matrices, and we are dealing with the parabolic equation

$$
\partial_{t} v(t, x)+H[v](t, x):=\partial_{t} v(t, x)+H\left(v(t, x), D v(t, x), D^{2} v(t, x), t, x\right)=0
$$

in subdomains of $(0, T) \times \mathbb{R}^{d}$, where $T \in(0, \infty)$,

$$
\begin{gathered}
\mathbb{R}^{d}=\left\{x=\left(x_{1}, \ldots, x_{d}\right): x_{1}, \ldots, x_{d} \in \mathbb{R}\right\}, \\
\partial_{t}=\frac{\partial}{\partial t}, \quad D^{2} u=\left(D_{i j} u\right), \quad D u=\left(D_{i} u\right), \quad D_{i}=\frac{\partial}{\partial x_{i}}, \quad D_{i j}=D_{i} D_{j} .
\end{gathered}
$$

Our main goal is to establish the existence of $L_{p}$-viscosity solutions of boundary value problems associated with (1.1), solutions, which are in $C^{1+\alpha}$ for an $\alpha \in(0,1)$.

The author was partially supported by NSF Grant DMS-1160569. 
Let us briefly discuss what the author was able to find in the literature concerning this kind of regularity. The articles cited below contain a very large amount of information concerning all kinds of issues in the theory of fully nonlinear elliptic and parabolic equations, but we will focus only on one of them.

Trudinger [15,16] and Caffarelli [2] were the first authors who proved $C^{1+\alpha}$ regularity for fully nonlinear elliptic equations of type

$$
F\left(u, D u, D^{2} u, x\right)=f
$$

without convexity assumptions on $F$. The assumptions in these papers are different. In [2] the function $F$ is independent of $u^{\prime}$ and, for each $u^{\prime \prime}$ uniformly sufficiently close to a function which is continuous with respect to $x$. In $[15,16]$ the function $F$ depends on all arguments but is Hölder continuous in $\left(u_{0}^{\prime}, x\right)$. Next step in what concerns $C^{1+\alpha}$-estimates for the elliptic case was done by Święch [14], who considered general $F$, imposed the same condition as in [2] on the $x$-dependence, which is much weaker than in $[15,16]$, but also imposed the Lipschitz condition on the dependence of $F$ on $u_{1}^{\prime}, \ldots, u_{d}^{\prime}$. In $[15,16]$ only continuity with respect to $u_{1}^{\prime}, \ldots, u_{d}^{\prime}$ is assumed.

In case of parabolic equations interior $C^{1+\alpha}$-regularity was established by Wang [17] under the same kind of assumption on the dependence of $H$ on $(t, x)$ as in [2] and assuming that $H$ is almost independent of $u_{1}^{\prime}, \ldots, u_{d}^{\prime}$. Then Crandall et al. [4] generalized the result of [17] to the case of full equation again as in [14] assuming that $H$ is uniformly sufficiently close to a function which is continuous with respect to $(t, x)$ and assuming the Lipschitz continuity of $H$ with respect to $u_{1}^{\prime}, \ldots, u_{d}^{\prime}$ and the continuity with respect to $u_{0}^{\prime}$.

On the one hand, our class of equations is more narrow than the one in [4] because we require the "main" part of $H$, called $F$, be positive homogeneous of degree one. On the other hand, we do not require $H$ to be Lipschitz with respect to $u_{1}^{\prime}, \ldots, u_{d}^{\prime}$, the continuity with respect to $u^{\prime}$ suffices. Also we only need $F$ to be measurable in $t$ and VMO in $x$, say, independent of $x$ and measurable in $t$.

As the referee kindly pointed out, there are other papers devoted to proving $C^{1+\alpha}$-regularity. One can find references to part of them in [1], which generalizes some previous results, but not the corresponding result of [4], which, as far as the author of the present paper understands, is much stronger than the corresponding result of [1].

Our methods are absolutely different from the methods of the above cited articles. We do not use any ideas or facts from the theory of viscosity solutions. Instead we rely on the methodology brought into the theory of fully nonlinear equations by Safonov [12,13] and on an idea behind the proof of the main Lemma 4.3 inspired by a probabilistic interpretation of solutions of (1.1). We only focus on interior estimates of solutions in smooth domains leaving to the interested reader investigation of the same issues in nonsmooth domains or near the boundary of sufficiently regular ones. We also leave to the interested reader carrying our results over to elliptic equations. 
The article is organized as follows. Section 2 contains main results and some comments on them. In Sect. 3 we use a theorem from [11] to approximate the equations with $H$ and with its main part $F$ by those for which the solvability is known. In Sect. 4 we show that the approximate principal equation with $F$ admits solutions locally well approximated in the sup norm by affine functions. This is the most important part of the article. Section 5 contains estimates of $C^{1+\alpha}$-norms of the approximate equation with full $H$ and in Sect. 6 we give the proof of our main Theorem 2.1. The last Sect. 7 is actually an appendix, which we need in order to be able to represent positive homogeneous of order one functions depending on parameters, such as $F$, as supinf's of affine functions whose coefficients inherit the regularity properties of the original function with respect to the parameters.

The author is sincerely grateful to the referee for drawing his attention to a few relevant papers and other useful comments.

\section{Main results}

To state our main results, we introduce a few notation and assumptions. Fix a constant $\delta \in(0,1]$, and set

$$
\mathbb{S}_{\delta}=\left\{a \in \mathbb{S}: \delta|\xi|^{2} \leq a_{i j} \xi_{i} \xi_{j} \leq \delta^{-1}|\xi|^{2}, \quad \forall \xi \in \mathbb{R}^{d}\right\}
$$

where and everywhere in the article the summation convention is enforced.

Assumption 2.1. (i) The function $H(u, t, x)$ is measurable with respect to $(t, x)$ for any $u$ and Lipschitz continuous in $u^{\prime \prime}$ for every $u^{\prime},(t, x) \in \mathbb{R}^{d+1}$.

(ii) For any $(t, x)$, at all points of differentiability of $H(u, t, x)$ with respect to $u^{\prime \prime}$, we have $\left(H_{u_{i j}^{\prime \prime}}\right) \in \mathbb{S}_{\delta}$.

(iii) There is a function $\bar{H}(t, x)$ and a constant $K_{0} \geq 0$ such that

$$
\left|H\left(u^{\prime}, 0, t, x\right)\right| \leq K_{0}\left|u^{\prime}\right|+\bar{H}(t, x) \text {. }
$$

(iv) There is an increasing continuous function $\omega(r), r \geq 0$, such that $\omega(0)=0$ and

$$
\left|H\left(u^{\prime}, u^{\prime \prime}, t, x\right)-H\left(v^{\prime}, u^{\prime \prime}, t, x\right)\right| \leq \omega\left(\left|u^{\prime}-v^{\prime}\right|\right)
$$

for all $u, v, t$, and $x$.

In a sense condition (iv) implies (iii). We still keep both because the constants entering our results depend on $K_{0}$ and $\bar{H}$, but they are independent of $\omega$.

For $R \in(0, \infty)$ and $(t, x) \in \mathbb{R}^{d+1}$ introduce

$$
\begin{aligned}
& B_{R}=\left\{x \in \mathbb{R}^{d}:|x|<R\right\}, \quad B_{R}(x)=x+B_{R}, \\
& C_{R}=\left(0, R^{2}\right) \times B_{R}, \quad C_{R}(t, x)=(t, x)+C_{R} .
\end{aligned}
$$

For a Borel set $\Gamma$ in $\mathbb{R}^{d+1}$ by $|\Gamma|$ we denote its Lebesgue measure. Also for a function $f$ on $\Gamma$ we set

$$
f_{\Gamma} f(t, x) d x d t=\frac{1}{|\Gamma|} \int_{\Gamma} f(t, x) d x d t
$$


in case $\Gamma$ has a nonzero Lebesgue measure in $\mathbb{R}^{d+1}$. Similar notation is used in case of functions $f(x)$ on $\mathbb{R}^{d}$.

We fix a constant $R_{0} \in(0,1]$ and for $\kappa \in(0,2]$ and measurable $f(t, x)$ introduce

$$
f_{\kappa}=\sup _{R \leq R_{0}, t, x} R^{2-\kappa}\left(f_{C_{R}(t, x)}|f(s, y)|^{d+1} d y d s\right)^{1 /(d+1)} .
$$

Remark 2.1. By Hölder's inequality for $p \geq d+1$

$$
\left(f_{C_{R}(t, x)}|f(s, y)|^{d+1} d y d s\right)^{1 /(d+1)} \leq N R^{-(d+2) / p}\left(f_{\mathbb{R}^{d+1}}|f(s, y)|^{p} d y d s\right)^{1 / p},
$$

which shows that $f_{\kappa}<\infty$ if $f \in L_{p}\left(\mathbb{R}^{d+1}\right)$ and $\kappa \leq 2-(d+2) / p$. It is useful to observe that one can take $\kappa>1$ if $f \in L_{p}\left(\mathbb{R}^{d+1}\right)$ for $p>d+2$.

In the following assumption there are three objects $\kappa_{1}=\kappa(d, \delta) \in(1,2)$, any $\kappa \in\left(1, \kappa_{1}\right]$, and $\theta=\theta(\kappa, d, \delta) \in(0,1]$. The values of $\kappa_{1}$ and $\theta$ are specified later in the proof of Lemma 5.3.

Assumption 2.2. We have a representation

$$
H(u, t, x)=F\left(u^{\prime \prime}, t, x\right)+G(u, t, x) .
$$

(i) The functions $F$ and $G$ are measurable functions of their arguments.

(ii) For all values of the arguments

$$
|G(u, t, x)| \leq K_{0}\left|u^{\prime}\right|+\bar{H}(t, x)
$$

and there exists a $\kappa \in\left(1, \kappa_{1}\right]$ such that $\bar{H}_{\kappa}<\infty$.

(iii) The function $F$ is positive homogeneous of degree one with respect to $u^{\prime \prime}$, is Lipschitz continuous with respect to $u^{\prime \prime}$, and at all points of differentiability of $F$ with respect to $u^{\prime \prime}$ we have $F_{u^{\prime \prime}} \in \mathbb{S}_{\delta}$.

(iv) For any $R \in\left(0, R_{0}\right],(t, x) \in \mathbb{R}^{d+1}$, and $u^{\prime \prime} \in \mathbb{S}$ with $\left|u^{\prime \prime}\right|=1\left(\left|u^{\prime \prime}\right|:=\right.$ $\left.\left(\operatorname{tr} u^{\prime \prime} u^{\prime \prime}\right)^{1 / 2}\right)$, we have

$$
\theta_{R, t, x}:=f_{C_{R}(t, x)}\left|F\left(u^{\prime \prime}, s, y\right)-\bar{F}_{R, x}\left(u^{\prime \prime}, s\right)\right| d s d y \leq \theta,
$$

where

$$
\bar{F}_{R, x}\left(u^{\prime \prime}, s\right)=f_{B_{R}(x)} F\left(u^{\prime \prime}, s, y\right) d y
$$

Remark 2.2. Assumption 2.2 (ii) is stronger than Assumption 2.1 (iii) which is singled out for methodological purposes. Also note that $G$ depends on $u$ and not only on $u^{\prime}$, and this will be important in an application to the Isaacs equations.

Also observe that one can take $\theta=0$ in Assumption 2.2 (iv) if $F$ is independent of $x$.

Fix a $T \in(0, \infty)$ and for domains $\Omega \subset \mathbb{R}^{d}$ define

$$
\Omega_{T}=(0, T) \times \Omega, \quad \partial^{\prime} \Omega_{T}=\bar{\Omega}_{T} \backslash(\{0\} \times \Omega) .
$$


For $\kappa \in(0,1]$ and functions $\phi(t, x)$ on $\bar{\Omega}_{T}$ set

$$
\begin{aligned}
{[\phi]_{C^{\kappa}\left(\bar{\Omega}_{T}\right)} } & =\sup _{(t, x),(s, y) \in \bar{\Omega}_{T}} \frac{|\phi(t, x)-\phi(s, y)|}{|t-s|^{\kappa / 2}+|x-y|^{\kappa}}, \quad\|\phi\|_{C\left(\bar{\Omega}_{T}\right)}=\sup _{\bar{\Omega}_{T}}|\phi|, \\
\|\phi\|_{C^{\kappa}\left(\bar{\Omega}_{T}\right)} & =\|\phi\|_{C\left(\bar{\Omega}_{T}\right)}+[\phi]_{C^{\kappa}\left(\bar{\Omega}_{T}\right)} .
\end{aligned}
$$

For $\kappa \in(1,2]$ and sufficiently regular $\phi$ set

$$
\begin{aligned}
{[\phi]_{C^{\kappa}\left(\bar{\Omega}_{T}\right)} } & =\sup _{t, s \in[0, T], x \in \mathbb{R}^{d}} \frac{|\phi(t, x)-\phi(s, x)|}{|t-s|^{\kappa / 2}}+\sup _{x, y \in \bar{\Omega}, t \in[0, T]} \frac{|D \phi(t, x)-D \phi(t, y)|}{|x-y|^{\kappa-1}}, \\
\|\phi\|_{C^{\kappa}\left(\bar{\Omega}_{T}\right)} & =\|\phi\|_{C^{1}\left(\bar{\Omega}_{T}\right)}+[\phi]_{C^{\kappa}\left(\bar{\Omega}_{T}\right)} .
\end{aligned}
$$

The set of functions with finite norm $\|\cdot\|_{C^{\kappa}\left(\bar{\Omega}_{T}\right)}$ is denoted by $C^{\kappa}\left(\bar{\Omega}_{T}\right)$.

Remark 2.3. According to the above notation $C^{2}\left(\bar{\Omega}_{T}\right)$ is not what is usually meant. Therefore, we are going to use the symbol $W_{\infty}^{1,2}\left(\Omega_{T}\right) \cap C\left(\bar{\Omega}_{T}\right)$ instead for the space provided with norm $\|\cdot\|_{C^{2}\left(\bar{\Omega}_{T}\right)}$. One should keep this in mind when we consider all $\kappa \in(0,2]$ at once.

For sufficiently regular functions $\phi(t, x)$ we set

$$
H[\phi](t, x)=H\left(\phi(t, x), D \phi(t, x), D^{2} \phi(t, x), t, x\right) .
$$

Similarly we introduce $F[\phi]$ and other operators if we are given functions of $u, t, x$.

Everywhere below $\Omega$ is a bounded $C^{2}$ domain in $\mathbb{R}^{d}$ and $T \in(0, \infty)$. The following is the main result of the paper. According to [4] we say that a function $u(t, x)$ is an $L_{p}$-viscosity subsolution of (1.1) in $\Omega_{T}$ if for any $\left(t_{0}, x_{0}\right) \in \Omega_{T}$ and any $\phi \in W_{p, l o c}^{1,2}\left(\Omega_{T}\right)$ for which $u-\phi$ is continuous at $\left(t_{0}, x_{0}\right)$ and attains a local maximum at $\left(t_{0}, x_{0}\right)$, we have

$$
\lim _{r \downarrow 0} \operatorname{ess}_{C_{r}\left(t_{0}, x_{0}\right)}\left[\partial_{t} \phi(t, x)+H\left(u(t, x), D \phi(t, x), D^{2} \phi(t, x), t, x\right)\right] \geq 0 .
$$

In a natural way one defines $L_{p}$-viscosity supersolutions and calls a function an $L_{p^{-}}$-viscosity solution if it is an $L_{p}$-viscosity supersolution and an $L_{p^{-}}$ viscosity subsolution. The reader is referred to [4] for numerous properties of $L_{p}$-viscosity solutions. Recall that $\kappa \in\left(1, \kappa_{1}\right]$.

Theorem 2.1. Let $g \in W_{\infty}^{1,2}\left(\Omega_{T}\right) \cap C\left(\bar{\Omega}_{T}\right)$. Then there is a function $v \in$ $C_{\text {loc }}^{\kappa}\left(\Omega_{T}\right) \cap C\left(\bar{\Omega}_{T}\right)$ which, for any $p>d+2$, is an $L_{p}$-viscosity solution of the equation

$$
\partial_{t} v+H[v]=0
$$

in $\Omega_{T}$ with boundary condition $v=g$ on $\partial^{\prime} \Omega_{T}$.

Furthermore, for any $r, R \in\left(0, R_{0}\right]$ satisfying $r<R$ and $(t, x) \in \Omega_{T}$ such that $C_{R}(t, x) \subset \Omega_{T}$ we have

$$
[v]_{C^{\kappa}\left(C_{r}(t, x)\right)} \leq N(R-r)^{-\kappa} \sup _{C_{R}(t, x)}|v|+N \bar{H}_{\kappa},
$$

where $N$ depend only on $d, \delta, K_{0}$, and $\kappa$ (in particular, independent of the function $\omega)$. 
Remark 2.4. A typical example of applications of Theorem 2.1 arises in connection with the theory of stochastic differential games where the so-called Isaacs equations play a major role. To describe a particular case of these equations, assume that we are given countable sets $A$ and $B$ and, for each $\alpha \in A$ and $\beta \in B$, we have an $\mathbb{S}_{\delta}$-valued function $a^{\alpha \beta}(t, x)$ defined on $\mathbb{R}^{d+1}$ and a real-valued function $G^{\alpha \beta}\left(u^{\prime}, t, x\right)$ defined for $u^{\prime},(t, x) \in \mathbb{R}^{d+1}$. Suppose that these functions are measurable and Assumption 2.2 (ii) is satisfied with $G^{\alpha \beta}$ in place of $G$ for any $\alpha \in A$ and $\beta \in B$ (and $\bar{H}$ independent of $\alpha \in A$ and $\beta \in B$ ). Also suppose that Assumption 2.1 (iv) is satisfied with the same function $\omega$ and with $G^{\alpha \beta}$ in place of $H$ for any $\alpha \in A$ and $\beta \in B$. Finally, suppose that for any $R \in\left(0, R_{0}\right]$ and $(t, x) \in$ $\mathbb{R}^{d+1}$

$$
f_{C_{R}(t, x)} \sup _{\alpha \in A} \sup _{\beta \in B}\left|a^{\alpha \beta}(s, y)-\bar{a}_{R, x}^{\alpha \beta}(s)\right| d s d y \leq \theta,
$$

where

$$
\bar{a}_{R, x}^{\alpha \beta}(s)=f_{B_{R}} a^{\alpha \beta}(s, y) d y .
$$

Upon introducing

$$
\begin{aligned}
F\left(u^{\prime \prime}, t, x\right) & =\sup _{\alpha \in A} \inf _{\beta \in B} a_{i j}^{\alpha \beta}(t, x) u_{i j}^{\prime \prime}, \\
G(u, t, x) & =\sup _{\alpha \in A} \inf _{\beta \in B}\left[a_{i j}^{\alpha \beta}(t, x) u_{i j}^{\prime \prime}+G^{\alpha \beta}\left(u^{\prime}, t, x\right)\right]-F\left(u^{\prime \prime}, t, x\right)
\end{aligned}
$$

one easily sees that Theorem 2.1 is applicable to the equation

$$
\partial_{t} v+\sup _{\alpha \in A} \inf _{\beta \in B}\left[a_{i j}^{\alpha \beta}(t, x) D_{i j}^{2} v+G^{\alpha \beta}(v, D v, t, x)\right]=0 .
$$

This example is close to the one from the introduction in [4] and is more general, because $G^{\alpha \beta}$ are not assumed to be linear in $u^{\prime}$. On the other hand, we suppose that Assumption 2.2 (ii) is satisfied with $G^{\alpha \beta}$ in place of $G$ uniformly in $\alpha, \beta$. In the situation of [4] only

$$
\left(\sup _{\alpha \in A} \inf _{\beta \in B} G^{\alpha \beta}(0, \cdot, \cdot)\right)_{\kappa}<\infty
$$

is required.

Remark 2.5. Assumption 2.2 (iii), (iv) can be replaced with the following which turns out to be basically weaker (cf.( 3.3)): There exist countable sets $A$ and $B$ and functions $a^{\alpha \beta}(t, x)$ satisfying the conditions of Remark 2.4 and there are numbers $f^{\alpha \beta}$ [independent of $(t, x)$ ] such that

$$
F\left(u^{\prime \prime}, t, x\right)=\sup _{\alpha \in A} \inf _{\beta \in B}\left[a_{i j}^{\alpha \beta}(t, x) u_{i j}^{\prime \prime}+f^{\alpha \beta}\right] \quad \text { and } \quad F(0, t, x) \equiv 0 .
$$




\section{Auxiliary equations}

In the first result of this section only Assumption 2.1 is used. By Theorem 2.1 of [11] there exists a convex positive homogeneous of degree one function $P\left(u^{\prime \prime}\right)$ such that at all points of differentiability of $P$ with respect to $u^{\prime \prime}$ we have $P_{u^{\prime \prime}}\left(u^{\prime \prime}\right) \in \mathbb{S}_{\hat{\delta}}$, where $\hat{\delta}=\hat{\delta}(d, \delta) \in(0, \delta / 4)$ and such that the following fact holds in which by $P[v]$ we mean a differential operator constructed as in (2.1).

Theorem 3.1. Let Assumption 2.1 be satisfied and $\bar{H}$ be bounded. Let $K \geq 0$ be a fixed constant, $g \in W_{\infty}^{1,2}\left(\Omega_{T}\right) \cap C\left(\bar{\Omega}_{T}\right)$. Then the equation

$$
\partial_{t} v+\max (H[v], P[v]-K)=0
$$

in $\Omega_{T}$ with boundary condition $v=g$ on $\partial^{\prime} \Omega_{T}$ has a solution $v \in C\left(\bar{\Omega}_{T}\right) \cap$ $W_{\infty, \text { loc }}^{1,2}\left(\Omega_{T}\right)$. In addition,

$$
|v|,|D v|, \rho\left|D^{2} v\right|,\left|\partial_{t} v\right| \leq N\left(\sup _{\Omega_{T}} \bar{H}+K+\|g\|_{C^{1,2}\left(\Omega_{T}\right)}\right) \quad \text { in } \Omega_{T} \text { (a.e.), }
$$

where

$$
\rho=\rho(x)=\operatorname{dist}\left(x, \mathbb{R}^{d} \backslash \Omega\right)
$$

and $N$ is a constant depending only on $\Omega, T, K_{0}$, and $\delta$ (in particular, independent of $\omega)$.

Theorem 3.1 is applicable to the equation

$$
\partial_{t} u+\max (F[u], P[u]-K)=0,
$$

which we want to rewrite in a different form.

First we observe that if in Sect. 7 we take $B=\{0\} \times \mathbb{S}_{\delta}$, take a strictly convex open set $B_{0}^{\prime}$ in $\mathbb{S}$ such that $\mathbb{S}_{\delta} \subset B_{0}^{\prime} \subset \mathbb{S}_{\delta / 2}$, and set $B_{0}=\{0\} \times \bar{B}_{0}^{\prime}$, then by Theorem 7.2 we have

$$
F\left(u^{\prime \prime}, t, x\right)=\sup _{\alpha \in A_{1}} \inf _{\beta \in B} a_{i j}^{\alpha \beta}(t, x) u_{i j}^{\prime \prime}, \quad \bar{F}\left(u^{\prime \prime}, t\right)=\sup _{\alpha \in A_{1}} \inf _{\beta \in B} \bar{a}_{i j}^{\alpha \beta}(t) u_{i j}^{\prime \prime},
$$

where $A_{1}=\mathbb{S}$, for $\alpha \in A_{1}$ and $\beta=\left(0, \beta^{\prime}\right) \in B$,

$$
\begin{aligned}
a^{\alpha \beta}(t, x) & =\lambda^{\alpha \beta}(t, x) \beta^{\prime}+\left(1-\lambda^{\alpha \beta}(t, x)\right) G_{u^{\prime \prime}}(\alpha) \\
\bar{a}^{\alpha \beta}(t) & =\bar{\lambda}^{\alpha \beta}(t, x) \beta^{\prime}+\left(1-\bar{\lambda}^{\alpha \beta}(t, x)\right) G_{u^{\prime \prime}}(\alpha), \quad G\left(u^{\prime \prime}\right)=\sup _{\beta^{\prime} \in B_{0}^{\prime}} \beta_{i j}^{\prime} u_{i j}^{\prime \prime}, \\
\lambda^{\alpha \beta}(t, x) & =1 \wedge \frac{G(\alpha)-F(\alpha, t, x)}{G(\alpha)-\beta_{i j}^{\prime} \alpha_{i j}}\left(\frac{0}{0}=1\right),
\end{aligned}
$$

and $\bar{\lambda}^{\alpha \beta}(t)$ is defined similarly. From Sect. 7 we also know that, for a constant $\mu>0$, we have $G(\alpha)-\beta_{i j}^{\prime} \alpha_{i j} \geq \mu|\alpha|$ if $\beta=\left(0, \beta^{\prime}\right) \in B$ and $\alpha \in A_{1}$.

Next, since $P\left(u^{\prime \prime}\right)$ is positive homogeneous, convex, and $P_{u^{\prime \prime}} \in \mathbb{S}_{\hat{\delta}}$, there exists a closed set $A_{2} \subset \mathbb{S}_{\hat{\delta}}$ such that

$$
P\left(u^{\prime \prime}\right)=\sup _{\alpha \in A_{2}} \alpha_{i j} u_{i j}^{\prime \prime}
$$

For uniformity of notation introduce $\hat{A}$ as a disjoint union of $A_{1}$ and $A_{2}$ and for $\beta \in B$ and $\alpha \in A_{2}$ set

$$
a^{\alpha \beta}(t, x)=\bar{a}^{\alpha \beta}(t)=\alpha .
$$


Also for $\alpha \in \hat{A}$ and $\beta \in B$ introduce $\sigma^{\alpha \beta}(t, x)=\left[a^{\alpha \beta}(t, x)\right]^{1 / 2}, \bar{\sigma}^{\alpha \beta}(t)=$ $\left[\bar{a}^{\alpha \beta}(t)\right]^{1 / 2}$,

$$
L^{\alpha \beta} v(t, x)=a_{i j}^{\alpha \beta}(t, x) D_{i j} v(t, x), \quad \bar{L}^{\alpha \beta} v(t, x)=\bar{a}_{i j}^{\alpha \beta}(t) D_{i j} v(t, x) .
$$

Next we have the following which is essentially Remark 3.1 of [5] with the proof based on the positive homogeneity and Lipschitz continuity of $F$ with respect to $u^{\prime \prime}$.

Lemma 3.2. There is a function $\theta=\theta(\mu)=\theta(\mu, d, \delta)>0$ defined for $\mu>0$ such that Assumption 2.2 (i), (iii), (iv) being satisfied with this $\theta(\mu)$ implies that for any $R \in\left(0, R_{0}\right]$ and $(t, x) \in \mathbb{R}^{d+1}$

$$
f_{C_{R}(t, x)} \sup _{u^{\prime \prime} \neq 0} \frac{\left|F\left(u^{\prime \prime}, s, y\right)-\bar{F}_{R, x}\left(u^{\prime \prime}, s\right)\right|}{\left|u^{\prime \prime}\right|} d s d y \leq \mu .
$$
$\mathbb{R}^{d+1}$

Note that by Lemma 3.2 and Theorem 7.3 for any $R \in\left(0, R_{0}\right]$ and $(t, x) \in$

$$
\mu_{R, t, x}:=f_{C_{R(t, x)}} \sup _{\alpha \in \hat{A}, \beta \in B}\left|a^{\alpha \beta}(s, y)-\bar{a}^{\alpha \beta}(s)\right| d s d y \leq N \mu,
$$

where the constant $N$ depends only on $d$ and $\delta$. On $\mathbb{S}_{\hat{\delta}}$ the function $a^{1 / 2}$ is Lipschitz continuous and therefore (3.3) also holds if we replace $a$ with $\sigma$.

Finally, observe that Eq. (3.2) is easily rewritten as

$$
\partial_{t} u+\sup _{\alpha \in \hat{A}} \inf _{\beta \in B}\left[L^{\alpha \beta} u(t, x)+f_{K}^{\alpha \beta}\right]=0,
$$

where $f_{K}^{\alpha \beta}=-K I_{\alpha \in A_{2}}$.

\section{Main estimate for solutions of (3.2)}

Take $R, K \in(0, \infty)$ and $g \in W_{\infty}^{1,2}\left(C_{R}\right) \cap C\left(\bar{C}_{R}\right)$. By Theorem 3.1 there exists $u \in W_{\infty, l o c}^{1,2}\left(C_{R}\right) \cap C\left(\bar{C}_{R}\right)$ such that $u=g$ on $\partial^{\prime} C_{R}$ and Eq. (3.2) holds (a.e.) in $C_{R}$. By the maximum principle such $u$ is unique.

Here is the main result of this section.

Theorem 4.1. There exist constants $\kappa_{0} \in(1,2]$ and $N \in(0, \infty)$ depending only on $d$ and $\delta$ such that for each $r \in(0, R]$ one can find an affine function $\hat{u}=\hat{u}(x)$ for which

$|u-\hat{u}| \leq N\left(\mu_{R}^{\kappa /(6 d+6)} \vee \mu_{R}^{1 /(6 d+6)}\right)[g]_{C^{\kappa}\left(\bar{C}_{R}\right)} R^{\kappa}+N r^{\kappa_{0}}(R-r)^{-\kappa_{0}} \underset{\partial^{\prime} C_{R}}{\operatorname{OSc}}(g-\hat{g})$

in $\bar{C}_{r}$ for any $\kappa \in(0,2]$, where $\mu_{R}=\mu_{R, 0,0}$ and $\hat{g}=\hat{g}(x)$ is any affine function of $x$.

By using parabolic dilations one easily sees that one may take $R=1$. In that case we first prove a few auxiliary results. Introduce $\bar{u}$ as a unique solution of (3.2) (in $C_{1}$ ) with $\bar{F}$ in place of $F$ and the same boundary condition on $\partial^{\prime} C_{1}$. Below by $N$ with occasional subscripts we denote various constants depending only on $d$ and $\delta$. 
Lemma 4.2. Let $\kappa \in(0,2]$ and

$$
[g]_{C^{\kappa}\left(\bar{C}_{1}\right)}=1 .
$$

Then for any $\varepsilon>0$ there exists an infinitely differentiable function $g^{\varepsilon}$ on $\mathbb{R}^{d+1}$ such that in $\bar{C}_{1}$

$$
\left|g-g^{\varepsilon}\right| \leq N \varepsilon^{\kappa}, \quad\left|\partial_{t} g^{\varepsilon}\right|+\left|D^{2} g^{\varepsilon}\right|+\varepsilon\left|D^{3} g^{\varepsilon}\right|+\varepsilon\left|D \partial_{t} g^{\varepsilon}\right| \leq N \varepsilon^{\kappa-2} .
$$

Furthermore, for $w=u, \bar{u}$ in $\bar{C}_{1}$ we have

$$
\left|w(t, x)-g^{\varepsilon}(t, x)\right| \leq N \varepsilon^{\kappa-2}\left(1-|x|^{2}\right)^{\kappa / 2}+N \varepsilon^{\kappa} .
$$

Proof. The first assertion is well known and is obtained by first continuing $g(t, x)$ as a function of $t$ to $\mathbb{R}$ to become an even 2-periodic function, then continuing thus obtained function across $|x|=1$ almost preserving (4.1) in the whole space and then taking convolutions with $\delta$-like kernels.

Then, since $K \geq 0$, for $w^{\varepsilon}=u-g^{\varepsilon}$ we have

$$
\partial_{t} w^{\varepsilon}+\partial_{t} g^{\varepsilon}+\max \left[F\left(D^{2} w^{\varepsilon}+D^{2} g^{\varepsilon}\right), P\left(D^{2} w^{\varepsilon}+D^{2} g^{\varepsilon}\right)\right] \geq 0,
$$

which in light of (4.2) implies that

$$
\partial_{t} w^{\varepsilon}+\max \left(F\left[w^{\varepsilon}\right], P\left[w^{\varepsilon}\right]\right) \geq-N_{1} \varepsilon^{\kappa-2} .
$$

Next, it is easily seen that there is a constant $N(=N(d, \delta))$ such that for $\phi^{\varepsilon}(t, x)=N N_{1} \varepsilon^{\kappa-2}\left(1-|x|^{2}\right)$ we have

$$
\partial_{t} \phi^{\varepsilon}+\max \left(F\left[\phi^{\varepsilon}\right], P\left[\phi^{\varepsilon}\right]\right) \leq-N_{1} \varepsilon^{\kappa-2}
$$

in $C_{1}$. It follows by the parabolic Alexandrov maximum principle that in $C_{1}$

$$
w^{\varepsilon} \leq \phi^{\varepsilon}+\sup _{\partial^{\prime} C_{1}}\left(w^{\varepsilon}-\phi^{\varepsilon}\right), \quad u \leq g^{\varepsilon}+N \varepsilon^{\kappa-2}\left(1-|x|^{2}\right)+N \varepsilon^{\kappa} .
$$

On the other hand,

$$
\begin{aligned}
\partial_{t} w^{\varepsilon}+\partial_{t} g^{\varepsilon}+F\left(D^{2} w^{\varepsilon}+D^{2} g^{\varepsilon}\right) & \leq 0, \\
\partial_{t} w^{\varepsilon}+F\left[w^{\varepsilon}\right] & \leq N \varepsilon^{\kappa-2},
\end{aligned}
$$

and with perhaps different constant in the formula for $\phi^{\varepsilon}$

$$
w^{\varepsilon} \geq-\phi^{\varepsilon}+\inf _{\partial^{\prime} C_{1}}\left(\phi^{\varepsilon}+w^{\varepsilon}\right), \quad u \geq g^{\varepsilon}-N \varepsilon^{\kappa-2}\left(1-|x|^{2}\right)-N \varepsilon^{\kappa},
$$

which along with (4.4) yields (4.3) for $w=u$. The proof of (4.3) for $w=\bar{u}$ is identical and the lemma is proved.

Lemma 4.3. For any $\kappa \in(0,2]$ in $\bar{C}_{1}$ we have

$$
|u-\bar{u}| \leq N\left(\mu_{1}^{\kappa /(6 d+6)} \vee \mu_{1}^{1 /(d+1)}\right)[g]_{C^{\kappa}\left(\bar{C}_{1}\right)} .
$$

Proof. To simplify some formulas observe that if $[g]_{C^{\kappa}\left(\bar{C}_{1}\right)}=0$, then $g$ is an affine function of $x$ independent of $t$, so that $u=\bar{u}=g$ and we have nothing to prove. However, if $[g]_{C^{\kappa}\left(\bar{C}_{1}\right)}>0$, we can divide Eq. (3.2) by this quantity, and, since our assertion means, in particular, that $N$ in (4.5) is independent of $K$, we can reduce the general situation to the one in which (4.1) holds. Therefore, below we assume (4.1). 
On sufficiently regular functions $u(t, x, \bar{x}), t \in \mathbb{R}, x, \bar{x} \in \mathbb{R}^{d}$, introduce

$$
\Phi[u](t, x, \bar{x})=\sup _{\alpha \in \hat{A}} \inf _{\beta \in B}\left[\mathcal{L}^{\alpha \beta} u(t, x, \bar{x})+f_{K}^{\alpha \beta}\right],
$$

where

$$
\begin{aligned}
& \mathcal{L}^{\alpha \beta} u(t, x, \bar{x})=a_{i j}^{\alpha \beta}(t, x) D_{i j}^{x} u(t, x, \bar{x})+\hat{a}_{i j}^{\alpha \beta}(t, x, \bar{x}) D_{i j}^{x \bar{x}} u(t, x, \bar{x}) \\
& +\check{a}_{i j}^{\alpha \beta}(t, x, \bar{x}) D_{i j}^{\bar{x} x} u(t, x, \bar{x})+\bar{a}_{i j}^{\alpha \beta}(t) D_{i j}^{\bar{x}} u(t, x, \bar{x}), \\
& D_{i j}^{x}=\frac{\partial^{2}}{\partial x_{i} \partial x_{j}}, \quad D_{i j}^{x \bar{x}}=\frac{\partial^{2}}{\partial x_{i} \partial \bar{x}_{j}}, \quad D_{i j}^{\bar{x} x}=\frac{\partial^{2}}{\partial \bar{x}_{i} \partial x_{j}}, \quad D_{i j}^{\bar{x}}=\frac{\partial^{2}}{\partial \bar{x}_{i} \partial \bar{x}_{j}}, \\
& \hat{a}^{\alpha \beta}(t, x, \bar{x})=\sigma^{\alpha \beta}(t, x) \bar{\sigma}^{\alpha \beta}(t), \quad \check{a}^{\alpha \beta}(t, x, \bar{x})=\bar{\sigma}^{\alpha \beta}(t) \sigma^{\alpha \beta}(t, x) .
\end{aligned}
$$

Observe that for $\lambda, \bar{\lambda} \in \mathbb{R}^{d}$ we have

$$
a_{i j}^{\alpha \beta} \lambda_{i} \lambda_{j}+\hat{a}_{i j}^{\alpha \beta} \lambda_{i} \bar{\lambda}_{j}+\check{a}_{i j}^{\alpha \beta} \bar{\lambda}_{i} \lambda_{j}+\bar{a}_{i j}^{\alpha \beta} \bar{\lambda}_{i} \bar{\lambda}_{j}=\left|\sigma^{\alpha \beta} \lambda+\bar{\sigma}^{\alpha \beta} \bar{\lambda}\right|^{2} \geq 0,
$$

so that $\Phi$ is a (degenerate) elliptic operator.

Next let $w \in W_{d+1}^{1,2}\left(C_{1}\right) \cap C\left(\bar{C}_{1}\right)$ be a solution of the equation

$$
\partial_{t} w+\sup _{\alpha \in \hat{A}} \sup _{\beta \in B} L^{\alpha \beta} w=-\sup _{\alpha \in A} \sup _{\beta \in B}\left|a^{\alpha \beta}-\bar{a}^{\alpha \beta}\right|=:-h
$$

in $C_{1}$ with zero boundary condition on $\partial^{\prime} C_{1}$. Such a unique solution exists by Theorem 1.1 of [5] and by the parabolic Alexandrov estimate and (3.3) we have in $C_{1}$ that

$$
0 \leq w \leq N \mu_{1}^{1 /(d+1)} .
$$

One of reasons we need the function $w$ for is that, as is easy to see, there is a $\lambda>0$ depending only on $d$ and $\delta$ such that for all $\alpha, \beta$ on $C_{1}$ we have

$$
\begin{gathered}
\partial_{t}\left(\lambda w(t, x)+|x-\bar{x}|^{2}\right)+\mathcal{L}^{\alpha \beta}(t, x, \bar{x})\left(\lambda w(t, x)+|x-\bar{x}|^{2}\right) \\
=\lambda\left(\partial_{t} w+L^{\alpha \beta} w\right)(t, x)+2\left|\sigma^{\alpha \beta}(t, x)-\sigma^{\alpha \beta}(t)\right|^{2} \leq 0,
\end{gathered}
$$

where the inequality follows from the fact that $a^{1 / 2}$ is a Lipschitz continuous function on $\mathbb{S}_{\hat{\delta}}$, so that $\left|\sigma^{\alpha \beta}-\bar{\sigma}^{\alpha \beta}\right|^{2} \leq N\left|a^{\alpha \beta}-\bar{a}^{\alpha \beta}\right|^{2} \leq N\left|a^{\alpha \beta}-\bar{a}^{\alpha \beta}\right|$.

After that we proceed in two steps.

Step 1. Estimate of $u-\bar{u}$ from above. According to Lemma 4.2 for $|\bar{x}|=1$ and $|x| \leq 1$ we have

$$
\begin{aligned}
u(t, x) & \leq g^{\varepsilon}(t, x)+N \varepsilon^{\kappa-2}\left(1-|x|^{2}\right)+N \varepsilon^{\kappa} \\
& \leq g^{\varepsilon}(t, x)+N \varepsilon^{\kappa-2}|x-\bar{x}|+N \varepsilon^{\kappa}
\end{aligned}
$$

where

$$
\varepsilon^{\kappa-2}|x-\bar{x}| \leq \varepsilon^{\kappa-4}|x-\bar{x}|^{2}+\varepsilon^{\kappa},
$$

so that

$$
u(t, x) \leq g^{\varepsilon}(t, x)+N \varepsilon^{\kappa-4}|x-\bar{x}|^{2}+N \varepsilon^{\kappa} .
$$

This inequality also obviously holds if $|x|=1,|\bar{x}| \leq 1$ or if $t=1$ and $|x|,|\bar{x}| \leq 1$. This shows that for $\varepsilon \in(0,1)$

$$
u^{\varepsilon}(t, x, \bar{x}):=u(t, x)-\left[g^{\varepsilon}(t, x)-g^{\varepsilon}(t, \bar{x})+N \varepsilon^{\kappa-6} e^{1-t}|x-\bar{x}|^{2}+N \varepsilon^{\kappa}\right] \leq g^{\varepsilon}(t, \bar{x})
$$


on $\partial^{\prime}\left[(0,1) \times C_{1}^{2}\right]$. Actually, above we could replace $\varepsilon^{\kappa-6}$ with $\varepsilon^{\kappa-4}$ but later on we will need to deal with terms of order $\varepsilon^{\kappa-6}|x-\bar{x}|^{2}$ anyway. Also observe that for $\varepsilon \in(0,1)$,

$$
\begin{aligned}
I^{\varepsilon}(t, x, \bar{x}):= & \partial_{t} u^{\varepsilon}(t, x, \bar{x})+\Phi\left[u^{\varepsilon}\right](t, x, \bar{x})=\partial_{t} u(t, x)+\partial_{t} g^{\varepsilon}(t, \bar{x})-\partial_{t} g^{\varepsilon}(t, x) \\
& +N \varepsilon^{\kappa-6} e^{1-t}|x-\bar{x}|^{2}+\sup _{\alpha \in \hat{A}} \inf _{\beta \in B}\left[a_{i j}^{\alpha \beta} D_{i j} u(t, x)+\bar{a}_{i j}^{\alpha \beta} D_{i j} g^{\varepsilon}(t, \bar{x})\right. \\
& \left.-a_{i j}^{\alpha \beta} D_{i j} g^{\varepsilon}(t, x)-N \varepsilon^{\kappa-6} e^{1-t}\left|\sigma^{\alpha \beta}(t, x)-\bar{\sigma}^{\alpha \beta}(t)\right|^{2}+f_{K}^{\alpha \beta}\right],
\end{aligned}
$$

where

$$
\begin{aligned}
\left|\bar{a}_{i j}^{\alpha \beta} D_{i j} g^{\varepsilon}(t, \bar{x})-a_{i j}^{\alpha \beta} D_{i j} g^{\varepsilon}(t, x)\right| & \leq\left|\bar{a}^{\alpha \beta}(t)-a^{\alpha \beta}(t, x)\right|\left|D^{2} g^{\varepsilon}(t, x)\right| \\
+N\left|D^{2} g^{\varepsilon}(t, x)-D^{2} g^{\varepsilon}(t, \bar{x})\right| & \leq N \varepsilon^{\kappa-2} h(t, x)+N \varepsilon^{\kappa-3}|x-\bar{x}|, \\
\left|\partial_{t} g^{\varepsilon}(t, \bar{x})-\partial_{t} g^{\varepsilon}(t, x)\right| & \leq N \varepsilon^{\kappa-3}|x-\bar{x}|, \\
-N \varepsilon^{\kappa-3}|x-\bar{x}|+N \varepsilon^{\kappa-6}|x-\bar{x}|^{2} & \geq-N \varepsilon^{\kappa} .
\end{aligned}
$$

It follows that for $\varepsilon \in(0,1)$,

$$
\begin{aligned}
I^{\varepsilon}(t, x, \bar{x}) & \geq \partial_{t} u(t, x)+\sup _{\alpha \in \hat{A}} \inf _{\beta \in B}\left[L^{\alpha \beta} u(t, x)+f_{K}^{\alpha \beta}\right]-N \varepsilon^{\kappa-6} h-N \varepsilon^{\kappa} \\
& =-N_{1} \varepsilon^{\kappa-6} h-N_{1} \varepsilon^{\kappa}
\end{aligned}
$$

in $(0,1) \times C_{1}^{2}$.

On the other hand,

$$
\bar{u}(t, \bar{x}) \geq g^{\varepsilon}(t, \bar{x})-N \varepsilon^{\kappa-2}\left(1-|\bar{x}|^{2}\right)-N \varepsilon^{\kappa},
$$

which implies that

$$
\begin{aligned}
\bar{u}^{\varepsilon}(t, x, \bar{x}) & :=\bar{u}(t, \bar{x})+N_{2} \varepsilon^{\kappa-6}\left((1+\lambda) w(t, x)+|x-\bar{x}|^{2}\right)+N_{2}(2-t) \varepsilon^{\kappa} \\
& \geq g^{\varepsilon}(t, \bar{x})
\end{aligned}
$$

on $\partial^{\prime}\left[(0,1) \times C_{1}^{2}\right]$. It is also easily seen that by increasing $N_{2}$ if needed (which does not violate the above inequality) we may assume that in $(0,1) \times C_{1}^{2}$

$$
\partial_{t} \bar{u}^{\varepsilon}(t, x, \bar{x})+\Phi\left[\bar{u}^{\varepsilon}\right](t, x, \bar{x}) \leq-N_{1} \varepsilon^{\kappa-6} h-N_{1} \varepsilon^{\kappa} .
$$

Hence, by the maximum principle (see, for instance, Theorem 2.1 of [8] or Theorem 3.4.2 of [9]) in $[0,1] \times \bar{C}_{1}^{2}$ we have

$$
\begin{aligned}
& \bar{u}(t, \bar{x})+N \varepsilon^{\kappa-6}\left(w(t, x)+|x-\bar{x}|^{2}\right)+N \varepsilon^{\kappa} \\
& \quad \geq u(t, x)-\left[g^{\varepsilon}(t, x)-g^{\varepsilon}(t, \bar{x})+N \varepsilon^{\kappa-6}|x-\bar{x}|^{2}+N \varepsilon^{\kappa}\right],
\end{aligned}
$$

which for $x=\bar{x}$ in light of (4.6) yields

$$
u(t, x)-\bar{u}(t, \bar{x}) \leq N\left(\varepsilon^{\kappa}+\varepsilon^{\kappa-6} \mu_{1}^{1 /(d+1)}\right) .
$$

If $\mu_{1} \leq 1$, then for $\varepsilon=\mu_{1}^{1 /(6 d+6)}(\leq 1)$ we get $u-\bar{u} \leq N \mu_{1}^{\kappa /(6 d+6)}$ and if $\mu_{1} \geq 1$, then for $\varepsilon=1$ we obtain $u-\bar{u} \leq N \mu_{1}^{1 /(d+1)}$, so that generally

$$
u-\bar{u} \leq N\left(\mu_{1}^{\kappa /(6 d+6)} \vee \mu_{1}^{1 /(d+1)}\right) .
$$


Step 2. Estimate of $u-\bar{u}$ from below. Notice that

$\bar{v}^{\varepsilon}(t, x, \bar{x}):=\bar{u}(t, \bar{x})-N \varepsilon^{\kappa-4}\left(\lambda w(t, x)+|x-\bar{x}|^{2}\right)-N \varepsilon^{\kappa} \leq g^{\varepsilon}(t, \bar{x})$

on $\partial^{\prime}\left[(0,1) \times C_{1}^{2}\right]$. It is also easily seen that in $(0,1) \times C_{1}^{2}$

$$
\partial_{t} \bar{v}^{\varepsilon}(t, x, \bar{x})+\Phi\left[\bar{v}^{\varepsilon}\right](t, x, \bar{x}) \geq 0 .
$$

On the other hand,

$$
\begin{aligned}
v^{\varepsilon}(t, x, \bar{x}):= & u(t, x)-\left[g^{\varepsilon}(t, x)-g^{\varepsilon}(t, \bar{x})\right]+N \varepsilon^{\kappa-6} e^{1-t}\left(\lambda w(t, x)+|x-\bar{x}|^{2}\right) \\
& +N(2-t) \varepsilon^{\kappa} \geq g^{\varepsilon}(t, \bar{x})
\end{aligned}
$$

on $\partial^{\prime}\left[(0,1) \times C_{1}^{2}\right]$ and the above computations show that (for sufficiently large $N$ )

$$
\partial_{t} v^{\varepsilon}(t, x, \bar{x})+\Phi\left[v^{\varepsilon}\right](t, x, \bar{x}) \leq 0
$$

in $(0,1) \times C_{1}^{2}$. By the maximum principle $\bar{v}^{\varepsilon} \leq v^{\varepsilon}$, which leads to the desired estimate of $u-\bar{u}$ from below and the lemma is proved.

Lemma 4.4. There exist constants $\kappa_{0} \in(1,2]$ and $N \in(0, \infty)$ depending only on $d$ and $\delta$ such that for any $r \in(0,1)$

$$
[\bar{u}]_{C^{\kappa_{0}}\left(\bar{C}_{r}\right)} \leq N(1-r)^{-\kappa_{0}} \underset{\partial^{\prime} C_{1}}{\operatorname{OSC}}(g-\hat{g}),
$$

where $\hat{g}=\hat{g}(x)$ is any affine function of $x$.

Proof. First observe that $\bar{u}-\hat{g}$ satisfies the same equation as $\bar{u}$ and the $C^{\kappa}\left(\bar{C}_{r}\right)$ seminorms of these functions coincide if $\kappa \in(1,2]$. It follows that we may concentrate on $\hat{g} \equiv 0$.

For unit $l \in \mathbb{R}^{d}$ and $h>0$ define $\delta_{l, h} \bar{u}(t, x)=h^{-1}[u(t, x+h l)-u(t, x)]$ and note that for any $\rho \in(0,1)$ the function $\delta_{l, h} \bar{u}$ satisfies an equation of type

$$
\partial_{t} \delta_{l, h} \bar{u}+a_{i j} D_{i j} \delta_{l, h} \bar{u}=0
$$

in $C_{\rho}$ with some $\left(a_{i j}\right)$ taking values in $\mathbb{S}_{\hat{\delta}}$ if $h$ is sufficiently small. By Corollary 4.3.6 of [9] for such $h$ and $r \in(0, \rho)$ we have

$$
\left[\delta_{l, h} \bar{u}\right]_{C \gamma\left(\bar{C}_{r}\right)} \leq N(\rho-r)^{-\gamma} \sup _{\bar{C}_{\rho}}\left|\delta_{l, h} \bar{u}\right|,
$$

where $N$ and $\gamma \in(0,1)$ depend only on $\delta$ and $d$. By letting $h \rightarrow 0$ we conclude

$$
[D \bar{u}]_{C^{\gamma}\left(\bar{C}_{r}\right)} \leq N(\rho-r)^{-\gamma} \sup _{\bar{C}_{\rho}}|D \bar{u}| .
$$

Next observe that for any function $f(x)$ of one variable $x \in[0, \varepsilon], \varepsilon>0$, we have

$$
\left|f^{\prime}(0)\right| \leq\left|f^{\prime}(0)-(f(\varepsilon)-f(0)) / \varepsilon\right|+\varepsilon^{-1} \underset{[0, \varepsilon]}{\operatorname{osc}} f \leq \varepsilon^{\gamma}\left[f^{\prime}\right]_{C \gamma[0, \varepsilon]}+\varepsilon^{-1} \underset{[0, \varepsilon]}{\operatorname{osc}} f .
$$

By applying this fact to functions $v(x)$ given in $B_{1}$ we obtain that for any $r_{n+1}<r_{n+2} \leq 1$ and any $\varepsilon \in(0,1)$

$$
|D v| \leq \varepsilon^{\gamma}\left(r_{n+2}-r_{n+1}\right)^{\gamma}[D v]_{C^{\gamma}\left(\bar{B}_{r_{n+2}}\right)}+\varepsilon^{-1}\left(r_{n+2}-r_{n+1}\right)^{-1} \underset{\bar{B}_{1}}{\operatorname{osc}} v
$$
in $\bar{B}_{r_{n+1}}$. 
Coming back to (4.8) and setting

$$
r_{0}=r, \quad r_{n}=r+(1-r) \sum_{k=1}^{n} 2^{-k}, \quad n \geq 1,
$$

we conclude

$$
\begin{aligned}
& A_{n}:=\sup _{\left[0, r_{n}^{2}\right]}[D \bar{u}(t, \cdot)]_{C^{\gamma}\left(\bar{B}_{r_{n}}\right)} \leq N\left(r_{n+1}-r_{n}\right)^{-\gamma} \sup _{\bar{C}_{r_{n+1}}}|D \bar{u}| \\
& \leq N_{1} \varepsilon^{\gamma} A_{n+2}+N_{2}(1-r)^{-(1+\gamma)} \varepsilon^{-1} 2^{(1+\gamma) n} \underset{\bar{C}_{1}}{\operatorname{Osc}} \bar{u}
\end{aligned}
$$

where the constants $N_{i}$ are different from the one in (4.8) but still depend only on $\delta$ and $d$. We first take $\varepsilon$ so that

$$
N_{1} \varepsilon^{\gamma}=2^{-5}
$$

then take $n=2 k, k=0,1, \ldots$, multiply both parts of (4.10) by $2^{-5 k}$ and sum up with respect to $k$. Then upon observing that $(1+\gamma) 2 k \leq 4 k$ we get

$$
\sum_{k=0}^{\infty} A_{2 k} 2^{-5 k} \leq \sum_{k=1}^{\infty} A_{2 k} 2^{-5 k}+N(1-r)^{-(1+\gamma)} \sum_{k=0}^{\infty} 2^{-k} \underset{\bar{C}_{1}}{\operatorname{Osc}} \bar{u} .
$$

By canceling (finite) like terms we find

$$
\sup _{\left[0, r^{2}\right]}[D \bar{u}(t, \cdot)]_{C^{\gamma}\left(\bar{B}_{r}\right)} \leq N(1-r)^{-(1+\gamma)} \operatorname{osc}_{\bar{C}_{1}} \bar{u} .
$$

Next, we use the fact that $\bar{u}$ itself satisfies the equation

$$
0=\partial_{t} \bar{u}+\max (\bar{F}[\bar{u}], P[\bar{u}]-K)-\max (0,-K)=\partial_{t} \bar{u}+a_{i j} D_{i j} \bar{u}
$$

with some $\left(a_{i j}\right)$ taking values in $\mathbb{S}_{\hat{\delta}}$. Furthermore, for any $T \in\left(0, r^{2}\right]$ and $\left|x_{0}\right| \leq r$ the function $v(t, x):=\bar{u}(t, x)-\left(x_{i}-x_{0 i}\right) D_{i} \bar{u}\left(T, x_{0}\right)$ satisfies the same equation and

$$
\left|v(T, x)-v\left(T, x_{0}\right)\right| \leq[D \bar{u}(T, \cdot)]_{C^{\gamma}\left(\bar{B}_{\rho}\right)}\left|x-x_{0}\right|^{1+\gamma}
$$

for $\left|x-x_{0}\right| \leq \rho-r$, where $\rho=(1+r) / 2$. Therefore, by Lemma 4.4.2 of [9], applied with $R=\rho-r=(1-r) / 2$ there, for $t \in[0, T]$ we have

$$
\begin{aligned}
& \left|\bar{u}\left(t, x_{0}\right)-\bar{u}\left(T, x_{0}\right)\right| \leq N[D \bar{u}(T, \cdot)]_{C \gamma\left(\bar{B}_{\rho}\right)}(T-t)^{(1+\gamma) / 2} \\
& \quad \leq N(1-r)^{-(1+\gamma)}(T-t)^{(1+\gamma) / 2} \underset{\bar{C}_{1}}{\operatorname{Osc}} \bar{u}
\end{aligned}
$$

This provides the necessary estimate of the oscillation of $\bar{u}$ in the time variable and along with (4.11) shows that

$$
[\bar{u}]_{C^{1+\gamma}\left(\bar{C}_{r}\right)} \leq N(1-r)^{-(1+\gamma)} \underset{\bar{C}_{1}}{\operatorname{osc}} \bar{u} .
$$

Now the assertion of the lemma follows from the fact that

$$
\underset{\bar{C}_{1}}{\operatorname{OSC}} \bar{u}=\underset{\partial^{\prime} C_{1}}{\operatorname{Osc}} g .
$$

The lemma is proved. 
Proof of Theorem 4.1. Take $\hat{u}(t, x)=\bar{u}(0,0)+x_{i} D_{i} \bar{u}(0,0)$ and observe that in $C_{r}$

$$
|u-\hat{u}| \leq|u-\bar{u}|+|\bar{u}-\hat{u}| \leq N\left(\mu_{1}^{\kappa /(6 d+6)} \vee \mu_{1}^{1 /(6 d+6)}\right)[g]_{C^{\kappa}\left(\bar{C}_{1}\right)}+I,
$$

where

$$
I=|\bar{u}-\hat{u}| \leq 2 r^{\kappa_{0}}[\bar{u}]_{C^{\kappa_{0}}\left(\bar{C}_{r}\right)} \leq N r^{\kappa_{0}}(1-r)^{-\kappa_{0}} \underset{\partial^{\prime} C_{1}}{\operatorname{OSC}}(g-\hat{g})
$$

so that the theorem is proved.

\section{Estimating $\boldsymbol{C}^{\boldsymbol{\kappa}}$-norm of solutions of (3.1)}

In this section we assume that $\bar{H}$ is bounded and investigate solutions of (3.1) which exist by Theorem 3.1. We take $\kappa_{0} \in(1,2]$ from Theorem 4.1 , take a $\mu \in(0,1]$, and suppose that Assumption 2.2 (iv) is satisfied with $\theta=\theta(\mu)$ so that (3.3) holds for any $R \in\left(0, R_{0}\right]$ and $(t, x) \in \mathbb{R}^{d+1}$.

Lemma 5.1. Let $R \in\left(0, R_{0}\right]$ and let $v \in W_{\infty}^{1,2}\left(C_{R}\right) \cap C\left(\bar{C}_{R}\right)$ be a solution of (3.1) in $\bar{C}_{R}$. Then for each $r \in(0, R)$ one can find an affine function $\hat{v}(x)$ such that in $C_{r}$ for any $\kappa \in[1,2]$

$$
\begin{aligned}
|v-\hat{v}| \leq & N \mu^{\kappa /(6 d+6)}[v]_{C^{\kappa}\left(\bar{C}_{R}\right)} R^{\kappa}+N r^{\kappa_{0}}(R-r)^{-\kappa_{0}} R^{\kappa}[v]_{C^{\kappa}\left(\bar{C}_{R}\right)} \\
& +N K_{0} R^{2} \sup _{\bar{C}_{R}}(|v|+|D v|)+N R^{\kappa} \bar{H}_{\kappa},
\end{aligned}
$$

where the constants $N$ depend only on $d$ and $\delta$.

Proof. Observe that

$$
\max (H[v], P[v]-K)=\max (F[v], P[v]-K)+h
$$

where $h$ defined by the above equality satisfies

$$
|h| \leq|H[v]-F[v]| \leq K_{0}(|v|+|D v|)+\bar{H} .
$$

Next define $u \in W_{d+1}^{1,2}\left(C_{R}\right) \cap C\left(\bar{C}_{R}\right)$ as a unique solution

$$
\partial_{t} u+\max (F[u], P[u]-K)=0
$$

with boundary data $u=v$ on $\partial^{\prime} C_{R}$. Then there exists an $\mathbb{S}_{\hat{\delta}}$-valued function $a$ such that in $C_{R}$ we have

$$
\partial_{t}(v-u)+a_{i j} D_{i j}(v-u)+h=0 .
$$

By the parabolic Alexandrov estimate (cf. our comment concerning this estimate in a more general situation in the proof of Lemma 6.1)

$$
\begin{aligned}
|v-u| & \leq N R^{d / d+1}\|h\|_{L_{d+1}\left(C_{R}\right)}=N R^{2}\left(f_{C_{R}}|h|^{d+1} d x d t\right)^{1 /(d+1)} \\
& \leq N K_{0} R^{2} \sup _{\bar{C}_{R}}(|v|+|D v|)+N R^{\kappa} \bar{H}_{\kappa} .
\end{aligned}
$$

After that our assertion follows from Theorem 4.1 and the lemma is proved. 
Here is a result, which can be easily extracted from the proof of Theorem 2.1 of [13].

Lemma 5.2. Let $r_{0} \in(0, \infty), \kappa \in(1,2), \phi \in C^{\kappa}\left(\bar{C}_{r_{0}}\right)$ and assume that there is a constant $N_{0}$ such that for any $(t, x) \in C_{r_{0}}$ and $r \in\left(0,2 r_{0}\right]$ there exists an affine function $\hat{\phi}=\hat{\phi}(x)$ such that

$$
\sup _{\bar{C}_{r}(t, x) \cap \bar{C}_{r_{0}}}|\phi-\hat{\phi}| \leq N_{0} r^{\kappa} .
$$

Then

$$
[\phi]_{C^{\kappa}\left(\bar{C}_{r_{0}}\right)} \leq N N_{0},
$$

where $N$ depends only on $d$ and $\kappa$.

Lemma 5.3. Take $r_{1} \in\left(0, R_{0}\right], r_{0} \in\left(0, r_{1}\right)$, and define

$$
\kappa_{1}=\frac{1+\kappa_{0}}{2} \text {. }
$$

Let $v \in W_{\infty}^{1,2}\left(C_{r_{1}}\right) \cap C\left(\bar{C}_{r_{1}}\right)$ be a solution of $(3.1)$ in $C_{r_{1}}$ and let $\kappa \in\left(1, \kappa_{1}\right]$. Then there exists $\theta=\theta(\kappa, d, \delta) \in(0,1]$ such that, if Assumption 2.2 (iv) is satisfied with this $\theta$, then

$$
\begin{aligned}
{[v]_{C^{\kappa}\left(\bar{C}_{r_{0}}\right) \leq} } & (1 / 2)[v]_{C^{\kappa}\left(\bar{C}_{r_{1}}\right)}+N\left(K_{0}+1\right)\left(r_{1}-r_{0}\right)^{-\kappa} \sup _{\bar{C}_{r_{1}}}|v| \\
& +N\left(K_{0}+1\right)\left(r_{1}-r_{0}\right)^{-(\kappa-1)} \sup _{\bar{C}_{r_{1}}}|D v|+N \bar{H}_{\kappa},
\end{aligned}
$$

where $N=N(d, \delta, \kappa)$.

Proof. To specify $\theta$ we first take a $\mu \in(0,1]$ and suppose that Assumption 2.2 (iv) is satisfied with $\theta=\theta(\mu)$ so that (3.3) holds for any $R \in\left(0, R_{0}\right]$ and $(t, x) \in \mathbb{R}^{d+1}$.

Then take $\left(t_{0}, x_{0}\right) \in C_{r_{0}}, \varepsilon \in(0,1)$, define

$$
r_{0}^{\prime}=\frac{\varepsilon}{3}\left(r_{1}-r_{0}\right)
$$

and notice that for any $(t, x) \in C_{r_{0}^{\prime}}\left(t_{0}, x_{0}\right), r \in\left(0,2 r_{0}^{\prime}\right]$, and $R=\varepsilon^{-1} r$, we have

$$
C_{R}(t, x) \subset C_{r_{1}} \text {. }
$$

Therefore, by Lemma 5.1 we can find an affine function $\hat{v}(x)$ such that

$$
\begin{aligned}
& \sup _{C_{r}(t, x) \cap C_{r_{0}^{\prime}}\left(t_{0}, x_{0}\right)}|v-\hat{v}| \leq \sup _{\bar{C}_{r}(t, x)}|v-\hat{v}| \leq N \mu^{\kappa /(6 d+6)}[v]_{C^{\kappa}\left(\bar{C}_{R}(t, x)\right)^{-\kappa}} r^{\kappa} \\
& \quad+N \varepsilon^{\kappa_{0}-\kappa}(1-\varepsilon)^{-\kappa} r^{\kappa}[v]_{C^{\kappa}\left(\bar{C}_{R}(t, x)\right)}+N K_{0} \varepsilon^{-2} r^{2} \sup _{\bar{C}_{R}(t, x)}(|v|+|D v|) \\
& \quad+N \varepsilon^{-\kappa} r^{\kappa} \bar{H}_{\kappa} \leq N r^{\kappa} I\left(\varepsilon, r_{1}\right),
\end{aligned}
$$

where the constants $N$ depend only on $d$ and $\delta$ and

$$
\begin{aligned}
I\left(\varepsilon, r_{1}\right):= & \left(\mu^{\kappa /(6 d+6)} \varepsilon^{-\kappa}+\varepsilon^{\kappa_{0}-\kappa}(1-\varepsilon)^{-\kappa_{0}}\right)[v]_{C^{\kappa}\left(\bar{C}_{r_{1}}\right)} \\
& +\varepsilon^{-2} K_{0} \sup _{\bar{C}_{r_{1}}}(|v|+|D v|)+\varepsilon^{-\kappa} \bar{H}_{\kappa} .
\end{aligned}
$$


It follows by Lemma 5.2 that

$$
[v]_{C^{\kappa}\left(\bar{C}_{r_{0}^{\prime}}\left(t_{0}, x_{0}\right)\right)} \leq N_{1} I\left(\varepsilon, r_{1}\right)
$$

where $N_{1}$ depends only on $d, \kappa$, and $\delta$. We can now specify $\theta$ and $\varepsilon$. First we chose $\varepsilon \in(0,1)$ so that

$$
N_{1} \varepsilon^{\kappa_{0}-\kappa}(1-\varepsilon)^{-\kappa_{0}}=1 / 4 .
$$

Since $\kappa_{0}-\kappa \geq\left(\kappa_{0}-1\right) / 2>0$ and $\kappa_{0}$ depends only on $d$ and $\delta$ and $N_{1}$ depends only on $d, \kappa$, and $\delta, \varepsilon$ also depends only on $d, \kappa$, and $\delta$. After that we take $\mu=\mu(d, \kappa, \delta) \in(0,1]$ so that

$$
N_{1} \mu^{1 /(6 d+6)} \varepsilon^{-2} \leq 1 / 4
$$

and set $\theta=\theta(\mu(d, \kappa, \delta))$. Then

$$
[v]_{C^{\kappa}\left(\bar{C}_{r_{0}^{\prime}}\left(t_{0}, x_{0}\right)\right)} \leq(1 / 2)[v]_{C^{\kappa}\left(\bar{C}_{r_{1}}\right)}+N J,
$$

where $N=N(d, \delta, \kappa)$ and

$$
J=K_{0} \sup _{\bar{C}_{r_{1}}}(|v|+|D v|)+\bar{H}_{\kappa} .
$$

Now observe that if $(t, x),(s, x) \in C_{r_{0}}$ and $t>s$, then either $|t-s| \leq$ $\left(r_{0}^{\prime}\right)^{2}$, in which case $(t, x) \in C_{r_{0}^{\prime}}(s, x)$ and

$$
(t-s)^{-\kappa / 2}|v(t, x)-v(s, x)| \leq(1 / 2)[v]_{C^{\kappa}\left(\bar{C}_{r_{1}}\right)}+N J
$$

owing to (5.2), or $|t-s| \geq\left(r_{0}^{\prime}\right)^{2}$ when $|v(t, x)-v(s, x)| \leq 2(t-s)^{\kappa / 2}\left(r_{0}^{\prime}\right)^{-\kappa} \sup _{\bar{C}_{r_{1}}}|v| \leq N(t-s)^{\kappa / 2}\left(r_{1}-r_{0}\right)^{-\kappa} \sup _{\bar{C}_{r_{1}}}|v|$.

Next if $(t, x),(t, y) \in C_{r_{0}}$ and $x \neq y$, then either $|x-y|<r_{0}^{\prime}$, in which case $(t, y) \in C_{r_{0}^{\prime}}(t, x)$ and

$$
|x-y|^{-(\kappa-1)}|D v(t, x)-D v(t, y)| \leq(1 / 2)[v]_{C^{\kappa}\left(\bar{C}_{r_{1}}\right)}+N J,
$$

or else $|x-y| \geq r_{0}^{\prime}$ and

$$
\begin{aligned}
|D v(t, x)-D v(t, y)| & \leq 2|x-y|^{\kappa-1}\left(r_{0}^{\prime}\right)^{-(\kappa-1)} \sup _{\bar{C}_{r_{1}}}|D v| \\
& \leq N|x-y|^{\kappa-1}\left(r_{1}-r_{0}\right)^{-(\kappa-1)} \sup _{\bar{C}_{r_{1}}}|D v| .
\end{aligned}
$$

This proves (5.1) and the lemma.

Theorem 5.4. Take $0<r<R \leq R_{0}$ and take $\kappa_{1}, \kappa \in\left(1, \kappa_{1}\right]$, and $\theta$ from Lemma 5.3. Let $v \in W_{\infty}^{1,2}\left(C_{R}\right) \cap \bar{C}\left(\bar{C}_{R}\right)$ be a solution of $(3.1)$ in $C_{R}$. Then

$$
[v]_{C^{\kappa}\left(\bar{C}_{r}\right)} \leq N(R-r)^{-\kappa} \sup _{\bar{C}_{R}}|v|+N \bar{H}_{\kappa},
$$

where $N$ depends only on $d, \delta, K_{0}$, and $\kappa$. 
Proof. We proceed as in the proof of Lemma 4.4. Fix a number $c \in(0,1)$ such that $c^{4}>3 / 4$ and introduce

$$
r_{0}=r, \quad r_{n}=r+c_{0}(R-r) \sum_{k=1}^{n} c^{k}, \quad n \geq 1,
$$

where $c_{0}$ is chosen in such a way that $r_{n} \rightarrow R$ as $n \rightarrow \infty$. Then Lemma 5.3 and (4.9) allow us to find constants $N_{1}$ and $N$ depending only on $d, \delta, K_{0}$, and $\kappa$ such that for all $n$ and $\varepsilon \in(0,1)$

$$
\begin{aligned}
A_{n}:= & {[v]_{C^{\kappa}\left(\bar{C}_{r_{n}}\right) \leq\left(2^{-1}+N_{1} \varepsilon^{\kappa-1}\right) A_{n+2}} } \\
& +N(R-r)^{-\kappa} c^{-n \kappa}\left(1+\varepsilon^{-1}\right) \sup _{\bar{C}_{R}}|v|+N \bar{H}_{\kappa} .
\end{aligned}
$$

We choose $\varepsilon<1$ so that $2^{-1}+N_{1} \varepsilon^{\kappa-1} \leq 3 / 4$ and then recalling that $\kappa \leq 2$ conclude that

$$
\begin{aligned}
\sum_{k=0}^{\infty}(3 / 4)^{k} A_{2 k} \leq & \sum_{k=1}^{\infty}(3 / 4)^{k} A_{2 k}+N \bar{H}_{\kappa} \\
& +N(R-r)^{-\kappa} \sup _{\bar{C}_{R}}|v| \sum_{k=0}^{\infty}(3 / 4)^{k} c^{-4 k},
\end{aligned}
$$

where the last series converges since $3 c^{-4} / 4<1$. By canceling like terms we come to (5.3) and the theorem is proved.

\section{Proof of Theorem 2.1}

First assume that $\bar{H}$ is bounded. For $K>0$ denote by $v_{K}$ the solution of (3.1) with boundary condition $v=g$ on $\partial^{\prime} \Omega_{T}$. By Theorem 3.1 such a solution exists is continuous in $\bar{\Omega}_{T}$ and has locally bounded derivatives.

Then the beginning of the proof of Lemma 5.1 shows that for an $\mathbb{S}_{\hat{\delta}}$-valued function $\left(a_{i j}\right)$ we have

$$
\left|\partial_{t} v_{K}+a_{i j} D_{i j} v_{K}\right| \leq K_{0}\left(\left|v_{K}\right|+\left|D v_{K}\right|+\bar{H}\right),
$$

and the parabolic Alexandrov estimate shows that

$$
\left|v_{K}\right| \leq N\left(\|g\|_{C\left(\Omega_{T}\right)}+\|\bar{H}\|_{L_{d+1}\left(\Omega_{T}\right)}\right)
$$

where $N$ depends only on $d, \delta, K_{0}$, and the diameter of $\Omega$.

Also

$$
\begin{aligned}
& \left|\partial_{t}\left(v_{K}-g\right)+a_{i j} D_{i j}\left(v_{K}-g\right)\right| \\
& \quad \leq K_{0}\left(\left|v_{K}\right|+\left|D\left(v_{K}-g\right)\right|\right)+\bar{H}+N\left(\left|\partial_{t} g\right|+\left|D^{2} g\right|+|D g|\right),
\end{aligned}
$$

which, after we continue $(v-g)(t, x)$ for $t \geq T$ as zero, by Theorem 4.2.6 of [9] yields that there exists an $\alpha=\alpha(d, \delta) \in(0,1)$ such that for any domain $\Omega^{\prime} \subset \bar{\Omega}^{\prime} \subset \Omega$

$$
\left|v_{K}\right|_{C^{\alpha}\left(\Omega_{T}^{\prime}\right)} \leq N
$$


where $N$ depends only on the distance between the boundaries of $\Omega^{\prime}$ and $\Omega$ and on $T, d, \delta, K_{0}$, the diameter of $\Omega$, and the $L_{d+1}\left(\Omega_{T}\right)$-norms of $\bar{H}$ and $\left|\partial_{t} g\right|+\left|D^{2} g\right|+|D g|$.

Now we are going to use one more piece of information available thanks to Theorem 2.1 of [11] which is that $v_{K} \in W_{p}^{1,2}\left(\Omega_{T}\right)$ for any $p$. Then treating (6.2) near $(0, T) \times \partial \Omega$ we can flatten $\partial \Omega$ near any given point, then continue $v-g$ (in the new coordinates) across the flat boundary in an odd way. We will then have a function of class $W_{d+1}^{1,2}$ to which Theorem 4.2.6 of [9] is applicable. In this way we estimate the $C^{\alpha}$-norm of $v$ near the boundary of $\Omega$ and in combination with (6.3) obtain that

$$
\left|v_{K}\right|_{C^{\alpha}\left(\bar{\Omega}_{T}\right)} \leq N_{0},
$$

where $N_{0}$ depends only on $T, d, \delta, K_{0}, \Omega$, and the $L_{d+1}\left(\Omega_{T}\right)$-norms of $\bar{H}$ and $\left|\partial_{t} g\right|+\left|D^{2} g\right|+|D g|$.

It follows that there is a sequence $K_{n} \rightarrow \infty$ and a function $v$ such that $v^{n}:=v_{K_{n}} \rightarrow v$ uniformly in $\bar{\Omega}_{T}$. Of course, (2.3) holds, owing to Theorem 5.4. Furthermore, (6.4) holds with the same constants and $v$ in place of $v_{K}$ and $D v^{n} \rightarrow D v$ locally uniformly in $\Omega_{T}$.

Next, we need an analog of Lemma 6.1 of [11]. Introduce

$$
H_{0}\left(u^{\prime \prime}, t, x\right)=H\left(v(t, x), D v(t, x), u^{\prime \prime}, t, x\right) .
$$

Lemma 6.1. There is a constant $N$ depending only on $d$ and $\delta$ such that for any $C_{r}(t, x)$ satisfying $C_{r}(t, x) \subset \Omega_{T}$ and $\phi \in W_{d+1}^{1,2}\left(C_{r}(t, x)\right) \cap C\left(\bar{C}_{r}(t, x)\right)$ we have on $C_{r}(t, x)$ that

$$
\begin{aligned}
& v \leq \phi+N r^{d /(d+1)}\left\|\left(\partial_{t} \phi+H_{0}[\phi]\right)^{+}\right\|_{L_{d+1}\left(C_{r}(t, x)\right)}+\max _{\partial^{\prime} C_{r}(t, x)}(v-\phi)^{+} . \\
& v \geq \phi-N r^{d /(d+1)}\left\|\left(\partial_{t} \phi+H_{0}[\phi]\right)^{-}\right\|_{L_{d+1}\left(C_{r}(t, x)\right)}-\max _{\partial^{\prime} C_{r}(t, x)}(v-\phi)^{-} .
\end{aligned}
$$

Proof. Observe that

$$
\begin{aligned}
& -\partial_{t} \phi-\max \left(H_{0}[\phi], P[\phi]-K_{n}\right) \\
& \quad=-\partial_{t} \phi-\max \left(H_{0}[\phi], P[\phi]-K_{n}\right)+\partial_{t} v^{n}+\max \left(H_{0}\left[v^{n}\right], P\left[v^{n}\right]-K_{n}\right)+I_{n} \\
& \quad=\partial_{t}\left(v^{n}-\phi\right)+a_{i j} D_{i j}\left(v^{n}-\phi\right)+I_{n},
\end{aligned}
$$

where $a=\left(a_{i j}\right)$ is an $\mathbb{S}_{\hat{\delta}}$-valued function and

$$
I_{n}=\max \left(H\left[v^{n}\right], P\left[v^{n}\right]-K_{n}\right)-\max \left(H_{0}\left[v^{n}\right], P\left[v^{n}\right]-K_{n}\right) .
$$

It follows by Theorem 3.1 of [8] or Theorem 3.3.9 of [9] that for $r \in(0,1]$

$$
\begin{aligned}
v^{n} \leq & \phi+\max _{\partial^{\prime} C_{r}(t, x)}\left(v^{n}-\phi\right)^{+} \\
& +N r^{d /(d+1)}\left\|\left(\partial_{t} \phi+I_{n}+\max \left(H[\phi], P[\phi]-K_{n}\right)\right)^{+}\right\|_{L_{d+1}\left(C_{r}(t, x)\right)},
\end{aligned}
$$

where the constant $N=N(d, \delta)$. Actually the above references only say that (6.7) holds with $N=N(r, d, \delta)$ in place of $N r^{d /(d+1)}$. However, the way this constant depends on $r$ is easily discovered by using parabolic dilations. 
Notice that

$$
\sup _{\bar{C}_{r}(t, x)}\left|I_{n}\right| \leq \omega\left(\sup _{\bar{C}_{r}(t, x)}\left(\left|v-v^{n}\right|+\left|D v-D v^{n}\right|\right)\right) \rightarrow 0
$$

as $n \rightarrow \infty$. After that we obtain (6.5) from (6.7) by letting $n \rightarrow \infty$. In the same way (6.6) is established. The lemma is proved.

After that the proof of Theorem 2.1 in our particular case of bounded $\bar{H}$ is achieved in the following way. Using (6.5) and repeating the proof of Theorem 2.3 of [11] (see Section 6 there), we easily obtain that, if $\left(t_{0}, x_{0}\right) \in \Omega_{T}$ and $\phi \in W_{d+1, \text { loc }}^{1,2}\left(\Omega_{T}\right)$ are such that $v-\phi$ attains a local maximum at $\left(t_{0}, x_{0}\right)$ and $v\left(t_{0}, x_{0}\right)=\phi\left(t_{0}, x_{0}\right)$, then

$$
\lim _{r \downarrow 0} \operatorname{ess} \sup _{C_{r}\left(t_{0}, x_{0}\right)}\left[\partial_{t} \phi(t, x)+H\left(v(t, x), D v(t, x), D^{2} \phi(t, x), t, x\right)\right] \geq 0 .
$$

Here $D v(t, x)$ can be replaced with $D v\left(t_{0}, x_{0}\right)$. Furthermore, if $\phi \in W_{p, l o c}^{1,2}\left(\Omega_{T}\right)$ with $p>d+2$, then by embedding theorems $\phi \in C_{l o c}^{1+\alpha}\left(\Omega_{T}\right)$, where $\alpha \in(0,1)$, and hence

$$
D v\left(t_{0}, x_{0}\right)=D \phi\left(t_{0}, x_{0}\right)
$$

It follows that one can replace $D v(t, x)$ with $D \phi(t, x)$ in $(6.8)$ and then, by definition $v$, is an $L_{p}$-viscosity subsolution.

The fact that it is also an $L_{p}$-viscosity supersolution is proved similarly on the basis of (6.6).

In case of general $\bar{H}$ we introduce $u_{n}$ as the solutions found according to Theorem 2.1 of $(2.2)$ in $\Omega_{T}$ with

$$
H(u, t, x) I_{\bar{H}(t, x) \leq n}+F\left(u^{\prime \prime}, t, x\right) I_{\bar{H}(t, x)>n}=F\left(u^{\prime \prime}, t, x\right)+G(u, t, x) I_{\bar{H}(t, x) \leq n}
$$

in place of $H(u, t, x)$ and with the same boundary condition $u_{n}=g$ on $\partial^{\prime} \Omega_{T}$. From the above we see that the estimates of $\left|u_{n}\right|_{C^{\alpha}\left(\bar{\Omega}_{T}\right)}$ and $\left[u_{n}\right]_{C^{\kappa}\left(\bar{C}_{r}(t, x)\right)}$ are uniform with respect to $n$. This allows us to repeat what was said about $v^{n}$ with obvious changes and brings the proof of Theorem 2.1 to an end.

\section{A minimax representation of nonlinear functions}

Here we complement the results of [10] which originated in [7] by providing a formula better suited for viewing nonlinear PDEs as Isaacs equations. In $[3,6]$ one can find other formulas applicable in more general situations, but they are of no use for us since they produce representations with a "free" term, which is (always) an unbounded function of control parameters $\alpha, \beta$.

Let $d_{1} \geq 1$ be an integer. Fix a closed bounded subset $B$ of $\mathbb{R}^{d_{1}+1}=$ $\left\{(f, l): f \in \mathbb{R}, l \in \mathbb{R}^{d_{1}}\right\}$. Let $H(u)$ be a real-valued Lipschitz continuous function given on $\mathbb{R}^{d_{1}}$. As a Lipschitz continuous function $H$ is differentiable on a set $D_{H}^{\prime} \subset \mathbb{R}^{d_{1}}$ of full measure. We introduce 


$$
\mathcal{L}(H):=\left\{(H(u)-\langle u, D H(u)\rangle, D H(u)): u \in D_{H}^{\prime}\right\},
$$

where $\langle\cdot, \cdot\rangle$ is the scalar product in $\mathbb{R}^{d_{1}}$, and assume that $\mathcal{L}(H) \subset B$. Observe that for $u \in D_{H}^{\prime}$ we have

$$
H(u)-\langle u, D H(u)\rangle=\left.\frac{\partial}{\partial t}[t H(u / t)]\right|_{t=1},
$$

so that, owing to the boundedness of $B, H$ is boundedly inhomogeneous.

Here is Remark 2.1 of [10] (in which we correct an obvious misprint).

Theorem 7.1. Under the above assumptions we have on $\mathbb{R}^{d_{1}}$ that

$$
H(u)=\max _{y \in \mathbb{R}^{d}} \min _{\substack{(f, l) \in B, f+\langle l, y\rangle \geq H(y)}}[f+\langle l, u\rangle]
$$

and the sets $\{(f, l) \in B: f+\langle l, y\rangle \geq H(y)\}$ are nonempty and closed for any $y \in \mathbb{R}^{d}$.

Next, let $B_{0}$ be a relatively strictly convex closed bounded set in $\mathbb{R}^{d_{1}+1}$ such that $B_{0} \supset B$ and the distance between the relative boundaries of $B$ and $B_{0}$ is strictly positive. Then introduce $A:=\mathbb{R}^{d_{1}}$ and for $\alpha \in A$ define

$$
G(\alpha)=\sup _{(f, l) \in B_{0}}(f+\langle l, \alpha\rangle) .
$$

Obviously $G \geq H$.

To derive some quantitative relations, let $\mathcal{P}$ be the smallest hyperplane containing $B_{0}$, and let $\left(f_{0}, l_{0}\right)$ be a fixed point in $\mathcal{P}$. By using the assumption about the boundaries of $B$ and $B_{0}$, define $\Gamma$ as a closed (in the topology of $\left.\mathcal{P}-\left(f_{0}, l_{0}\right)\right)$ convex subset of $\mathcal{P}-\left(f_{0}, l_{0}\right)$ with the origin lying in the relative (in the topology of $\mathcal{P}-\left(f_{0}, l_{0}\right)$ ) interior of $\Gamma$ such that $(f, l)+\Gamma \subset B_{0}$ for any $(f, l) \in B$. Define

$$
\gamma(u)=\sup _{(f, l) \in \Gamma}[f+\langle l, u\rangle]
$$

and observe that since $\pm \mu\left(B_{0}-\left(f_{0}, l_{0}\right)\right) \subset \Gamma$ for a constant $\mu>0$ we have that

$$
\mu\left|G(\alpha)-f_{0}-\left\langle l_{0}, \alpha\right\rangle\right| \leq \gamma(\alpha), \quad \mu\left|H(\alpha)-f_{0}-\left\langle l_{0}, \alpha\right\rangle\right| \leq \gamma(\alpha) .
$$

Furthermore, since $(f, l)+\Gamma \subset B_{0}$ for any $(f, l) \in B$ we have that for any $(f, l) \in B$

$$
f+\langle l, \alpha\rangle+\gamma(\alpha) \leq \sup _{\left(f^{\prime}, l^{\prime}\right) \in B_{0}}\left(f^{\prime}+\left\langle l^{\prime}, \alpha\right\rangle\right), \quad G(\alpha)-[f+\langle l, \alpha\rangle] \geq \gamma(\alpha),
$$

which shows that

$$
\lambda_{H}^{\alpha \beta}:=1 \wedge \frac{G(\alpha)-H(\alpha)}{G(\alpha)-[f+\langle l, \alpha\rangle]} \quad\left(\frac{0}{0}=1\right)
$$

is well defined for $\alpha \in A$ and $\beta=(f, l) \in B$ and, of course, $\lambda_{H}^{\alpha \beta} \in[0,1]$.

Next, observe that the graph of

$$
G(\xi, \alpha):=\sup _{(f, l) \in B_{0}}(f \xi+\langle l, \alpha\rangle)
$$


is a cone with respect to $(\xi, \alpha)$ which is once continuously differentiable with respect to $(\lambda, \alpha)$ everywhere apart from the origin due to the strict convexity of $B_{0}$. Since the plane $\xi=1$ does not pass through the origin, $G(\alpha)$ is once continuously differentiable.

Now for $\alpha \in A$ and $\beta=(f, l) \in B$ set

$$
\begin{aligned}
f_{H}^{\alpha \beta} & =\lambda_{H}^{\alpha \beta} f+\left(1-\lambda_{H}^{\alpha \beta}\right)[G(\alpha)-\langle\alpha, D G(\alpha)\rangle], \\
l_{H}^{\alpha \beta} & =\lambda_{H}^{\alpha \beta} l+\left(1-\lambda_{H}^{\alpha \beta}\right) D G(\alpha) .
\end{aligned}
$$

Obviously $\left(f_{H}^{\alpha \beta}, l_{H}^{\alpha \beta}\right) \in B_{0}$. In this way on the set $\mathcal{H}(B)$ of functions $H$ satisfying the assumptions stated in the beginning of the section we constructed a mapping sending each $H \in \mathcal{H}(B)$ into the function $\left(f_{H}^{\alpha \beta}, l_{H}^{\alpha \beta}\right)$ defined on $A \times B$.

Theorem 7.2. For any $H \in \mathcal{H}(B)$ and any $u \in \mathbb{R}^{d_{1}}$ we have

$$
H(u)=\sup _{\alpha \in A} \inf _{\beta \in B}\left[f_{H}^{\alpha \beta}+\left\langle l_{H}^{\alpha \beta}, u\right\rangle\right] .
$$

Furthermore, if $H, F \in \mathcal{H}(B)$, then for any $\alpha \in A$ and $\beta=(f, l) \in B$ we have

$$
\begin{aligned}
& \left|f_{H}^{\alpha \beta}-f_{F}^{\alpha \beta}\right| \leq \frac{|H(\alpha)-F(\alpha)|}{\gamma(\alpha)}|f+\langle\alpha, D G(\alpha)\rangle-G(\alpha)| \quad\left(\frac{0}{0}=0\right), \\
& \left|l_{H}^{\alpha \beta}-l_{F}^{\alpha \beta}\right| \leq \frac{|H(\alpha)-F(\alpha)|}{\gamma(\alpha)}|l-D G(\alpha)\rangle \mid \quad\left(\frac{0}{0}=0\right) .
\end{aligned}
$$

Proof. Observe that, for $\beta=(f, l) \in B$, if $f+\langle l, \alpha\rangle \geq H(\alpha)$, then $G(\alpha)-$ $H(\alpha) \geq G(\alpha)-[f+\langle l, \alpha\rangle]$ and $\lambda_{H}^{\alpha \beta}=1$ (no matter $\gamma(\alpha)=0$ or $\left.\gamma(\alpha)>0\right)$ and $f=f_{H}^{\alpha \beta}$ and $l=l_{H}^{\alpha \beta}$. It follows that

$$
\min _{\substack{\beta=(f, l) \in B, f+\langle l, \alpha\rangle \geq H(\alpha)}}[f+\langle l, u\rangle] \geq \inf _{\substack{\beta \in B, \lambda_{H}^{\alpha \beta}=1}}\left[f_{H}^{\alpha \beta}+\left\langle l_{H}^{\alpha \beta}, u\right\rangle\right] \geq \inf _{\beta \in B}\left(f_{H}^{\alpha \beta}+\left\langle l_{H}^{\alpha \beta}, u\right\rangle\right) .
$$

Furthermore, for $\beta=(f, l) \in B$, if $\lambda_{H}^{\alpha \beta}=1$, then $G(\alpha)-H(\alpha) \geq G(\alpha)-$ $[f+\langle l, \alpha\rangle],\left(f_{H}^{\alpha \beta}, l_{H}^{\alpha \beta}\right)=(f, l) \in B_{0}$, and

$$
f_{H}^{\alpha \beta}+\left\langle l_{H}^{\alpha \beta}, \alpha\right\rangle \geq H(\alpha) .
$$

In addition, for $\beta=(f, l) \in B$, if $\lambda_{H}^{\alpha \beta}<1$, then as always $\left(f_{H}^{\alpha \beta}, l_{H}^{\alpha \beta}\right) \in B_{0}$ and

$$
f_{H}^{\alpha \beta}+\left\langle l_{H}^{\alpha \beta}, \alpha\right\rangle=\lambda_{H}^{\alpha \beta}[f+\langle l, \alpha\rangle]+\left(1-\lambda_{H}^{\alpha \beta}\right) G(\alpha)=H(\alpha) .
$$

Hence,

$$
\min _{\substack{\beta=(f, l) \in B, f+\langle l, \alpha\rangle \geq H(\alpha)}}[f+\langle l, u\rangle] \geq \inf _{\beta \in B}\left(f_{H}^{\alpha \beta}+\left\langle l_{H}^{\alpha \beta}, u\right\rangle\right) \geq \min _{\substack{(f, l) \in B_{0}, f+\langle l, \alpha\rangle \geq H(\alpha)}}[f+\langle l, u\rangle],
$$

and the first assertion of the theorem follows from Theorem 7.1.

To prove the second assertion it suffices to note that, for instance,

$$
f_{H}^{\alpha \beta}-f_{F}^{\alpha \beta}=\left(\lambda_{H}^{\alpha \beta}-\lambda_{F}^{\alpha \beta}\right)[f+\langle\alpha, D G(\alpha)\rangle-G(\alpha)],
$$

where the right-hand side is zero if $\gamma(\alpha)=0$, and after that use (7.2). The theorem is proved. 
Theorem 7.3. Let $H$ also depend on parameters $(t, x) \in \mathbb{R}^{d+1}$ and let it satisfy the assumptions in the beginning of the section for each $(t, x)$. Assume that $H(u, t, x)$ is measurable with respect to $(t, x)$ and there is a function $\bar{H}(u, t)$ also satisfying the assumptions in the beginning of the section for each $t$ and measurable with respect to $t$. Denote

$$
\theta=\int_{C_{1,1}} \sup _{u: \gamma(u) \neq 0} \frac{|H(u, t, x)-\bar{H}(u, t)|}{\gamma(u)} d x d t .
$$

Also let $\left(f^{\alpha \beta}(t, x), l^{\alpha \beta}(t, x)\right)$ correspond to $H(u, t, x)$ and $\left(\bar{f}^{\alpha \beta}(t), \bar{l}^{\alpha \beta}(t)\right)$ correspond to $\bar{H}(u, t)$ constructed as before Theorem 7.2. Then

$$
\int_{C_{1,1}} \sup _{\alpha \in A, \beta \in B}\left(\left|f^{\alpha \beta}(t, x)-\bar{f}^{\alpha \beta}(t)\right|+\left|l^{\alpha \beta}(t, x)-\bar{l}^{\alpha \beta}(t)\right|\right) d x d t \leq N \theta,
$$

where the constant $N$ depends only on $B$ and $B_{0}$.

Proof. Let $\lambda^{\alpha \beta}(t, x)$ correspond to $H(u, t, x)$ and $\bar{\lambda}^{\alpha \beta}(t)$ correspond to $\bar{H}(u, t)$ constructed as before Theorem 7.2. Then it suffices to prove that

$$
\int_{C_{1,1}} \sup _{\alpha \in A, \beta \in B}\left|\lambda^{\alpha \beta}(t, x)-\bar{\lambda}^{\alpha \beta}(t)\right| d x d t \leq N \theta .
$$

For $\beta=(f, l) \in B$ we have

$$
\begin{aligned}
\left|\lambda^{\alpha \beta}(t, x)-\bar{\lambda}^{\alpha \beta}(x)\right| & \leq\left|\frac{G(\alpha)-H(\alpha, t, x)}{G(\alpha)-[f+\langle l, \alpha\rangle]}-\frac{G(\alpha)-\bar{H}(\alpha, t)}{G(\alpha)-[f+\langle l, \alpha\rangle]}\right| \\
& \leq \frac{|H(\alpha, t, x)-\bar{H}(\alpha, t)|}{\gamma(\alpha)}
\end{aligned}
$$

(with $0 / 0=0$ ) and our assertion follows. The theorem is proved.

\section{References}

[1] Bourgoing, M.: $C^{1, \beta}$ regularity of viscosity solutions via a continuous-dependence result. Adv. Differ. Equ. 9(3-4), 447-480 (2004)

[2] Caffarelli, L.: Interior a priori estimates for solutions of fully nonlinear equations. Ann. Math. 130, 189-213 (1989)

[3] Evans, L.C.: On solving certain nonlinear partial differential equations by accretive operator methods. Isr. J. Math. 36, 225-247 (1980)

[4] Crandall, M.G., Kocan, M., Święch, A.: $L^{p}$-theory for fully nonlinear uniformly parabolic equations. Commun. Partial Differ. Equ. 25(11-12), 1997-2053 (2000)

[5] Dong, H., Krylov, N.V., Li, X.: On fully nonlinear elliptic and parabolic equations in domains with VMO coefficients. Algebra Anal. 24(1), 54-95 (2012) in Russian; English translation in St. Petersb. Math. J. 24, 39-69 (2013)

[6] Katsoulakis, M.A.: A representation formula and regularizing properties for viscosity solutions of second-order fully nonlinear degenerate parabolic equations. Nonlinear Anal. 24(2), 147-158 (1995) 
[7] Krylov, N.V.: Bounded inhomogeneous nonlinear elliptic and parabolic equations in the plane. Mat. Sb. (N.S.) 82(1), 99-110 (1970) in Russian; English translation in Math. USSR Sb. 11(1), 89-99 (1970)

[8] Krylov, N.V.: On the maximum principle for nonlinear parabolic and elliptic equations. Izv. Akad. Nauk SSSR Ser. Mat. 42(5), 1050-1062 (1978) in Russian; English translation in Math. USSR Izv. 13(2), 335-347 (1979).

[9] Krylov, N.V.: Nonlinear elliptic and parabolic equations of second order. Nauka, Moscow (1985) in Russian; English translation: Reidel, Dordrecht (1987)

[10] Krylov, N.V.: On a representation of fully nonlinear elliptic operators in terms of pure second order derivatives and its applications. Probl. Math. Anal. 59, 3-24 (2011) in Russian; English translation in J. Math. Sci. N. Y. 177(1), 1-26 (2011).

[11] Krylov, N.V.: An ersatz existence theorem for fully nonlinear parabolic equations without convexity assumptions. http://arxiv.org/abs/1211.3732

[12] Safonov M.V.: On the classical solution of Bellman's elliptic equation. Dokl. Akad. Nauk SSSR 278(4), 810-813 (1984) in Russian; English translation in Sov. Math. Dokl. 30, 482-485 (1984)

[13] Safonov, M.V.: On the classical solution of nonlinear elliptic equations of second order. Izv. Akad. Nauk SSSR Ser. Mat. 137(2), 184-201 (1988) in Russian; English translation in Math. USSR Izv. 33(3), 597-612 (1989)

[14] Święch, A.: $W^{1, p}$-interior estimates for solutions of fully nonlinear, uniformly elliptic equations. Adv. Differ. Equ. 2(6), 1005-1027 (1997)

[15] Trudinger, N.S.: Hölder gradient estimates for fully nonlinear elliptic equations. Proc. R. Soc. Edinb. Sect. A 108(1-2), 57-65 (1988)

[16] Trudinger, N.S.: On regularity and existence of viscosity solutions of nonlinear second order, elliptic equations, pp. 939-957. In: Partial differential equations and the calculus of variations, vol. II. Progr. Nonlinear Differ. Equ. Appl., 2. Birkhäuser, Boston (1989)

[17] Wang, L.: On the regularity theory of fully nonlinear parabolic equations. II. Comm. Pure Appl. Math. 45(2), 141-178 (1992)

N. V. Krylov

University of Minnesota

127 Vincent Hall

Minneapolis

MN 55455

USA

e-mail: krylov@math.umn.edu

Received: 24 December 2012.

Accepted: 17 April 2013. 